\title{
Performance Analysis of an Optical CDMA Random Access Protocol
}

\author{
Hossam M. H. Shalaby, Senior Member, IEEE
}

\begin{abstract}
A random-access protocol for an optical direct-detection code-division-multiple-access (CDMA) packet network is proposed. This protocol is called a round-robin receiver/transmitter ( $\left.R^{3} T\right)$ protocol. A mathematical description of the protocol is presented using a detailed state diagram. Several performance measures, namely, steady-state system throughput, protocol efficiency, and average packet delay, are derived based on the equilibrium point-analysis technique. The effect of several design parameters on the above performance measures have been examined with the aid of a set of numerical examples. In our numerical calculations chip-level models have been adopted at the receiver side. Our results reveal that an efficiency of more than $90 \%$ can be reached easily with mild system parameters. Further an asymptotic efficiency of $\approx 100 \%$ is also achievable.
\end{abstract}

Index Terms-Chip-level receivers, code division multiple access (CDMA), direct-detection optical channel, on-off keying, optical CDMA, optical CDMA protocols, optical link layer, optical networks, random-access protocols.

\section{INTRODUCTION}

D UE to the vast bandwidth offered by the optical fibers and the extra-high optical signal processing speed bestowed by the optical components, optical code-division-multiple-access (CDMA) techniques have been given an increasing interest in the last decade [1]-[17]. In addition, optical CDMA technique has several advantages over other multiple access techniques, e.g., unnecessary time synchronization and frequency management, simple communication protocols, complete utilization of the entire time-frequency domain by each subscriber, flexibility in network design, and security against interception. Consequently, a large number of simultaneous users can be accommodated in local- and metropolitan-area networks (LANs and MANs) that employ optical CDMA techniques.

During the last and long period of research in the field of optical CDMA networks, however, most authors have focused on the physical layer of the communication network and only a few of them [13]-[17] have studied its network or link layer.

In [17], we have proposed two different protocols for slotted optical CDMA packet networks that needed pretransmission coordination. A variant of one of these two protocols that does not need pretransmission coordination has also been described in [17]. In our analysis of the performance of these protocols, we have oversimplified the system in order to have some insight on

Manuscript received August 5, 2003; revised January 31, 2004.

The author is with the Department of Electrical Engineering, Faculty of Engineering, University of Alexandria, Alexandria 21544, Egypt (e-mail: shalaby@ieee.org).

Digital Object Identifier 10.1109/JLT.2004.827659 the problem. Indeed we neither answered the following questions, nor did we take their effect on the performance evaluation in [17]:

1) how the transmitter deals with multipacket messages;

2) how the transmitter responds to an arrived message;

3) how the transmitter manages lost packets or packets received with errors:

4) how the receiver responds to a request for communication;

5) how the propagation delay and transmitter/receiver tuning time affect the network performance.

In this paper, we aim at answering the above questions and exploring their effect on the overall performance. To answer the above questions we first describe the detailed architecture of an optical CDMA network. Next we propose a new optical CDMA protocol that takes care of the above questions. To explore the effect of these issues on the network performance, we face a prohibitively large number of states, which makes the problem analytically intractable. Fortunately, the equilibrium point analysis (EPA) technique significantly simplifies the problem and makes it more tractable. In this technique, the system is always assumed to be operating at an equilibrium point [18]-[20]. That is at any time slot, the expected number of users entering any state is always equal to that departing from the state.

\section{A. System Architecture}

The basic architecture of our optical CDMA network is composed of a set of $N$ nodes or users, an optical star network, and a set of optical-orthogonal codes (OOCs) $C=\left\{a_{1}, a_{2}, a_{3}, \ldots, a_{|C|}\right\}$ with cardinality $|C|$. The cardinality $|C|$ depends on the code length $L$, the code weight $w$, and both the out-of-phase autocorrelation and cross-correlation constraints $\lambda_{a}, \lambda_{c}$, respectively. Traditionally $\lambda_{a}=\lambda_{c}=1$, which gives [1], [2]

$$
|C|=\left\lfloor\frac{L-1}{w(w-1)}\right\rfloor
$$

where $\lfloor x\rfloor$ denotes the largest integer not greater than $x$. Since under normal situations the network users send their data in a burst mode, i.e., they are not all active at the same time, we allow the total number of users to exceed the number of available codes, $N>|C|$. Codes are assigned to all users a priori. That is when a user subscribes to the network, it is given a code (possibly used) randomly from $C$. Furthermore, the code is randomly cyclic shifted around itself once assigned.

Each user is assigned a fixed CDMA encoder and a tunable CDMA decoder (FT-TR). The transmitter is able to generate an optical ON-OFF keying CDMA (OOK-CDMA) signal 
(according to its signature code) that represents its data. The tunable receiver is able to tune to any code of all other transmitters. The structure of OOK-CDMA tunable receivers has been thoroughly studied in [6]. Indeed, Zhang [6] has used a set of electrooptic switches in his receiver implementation. He has shown that for a code with length $L$ and weight $w$, only $(w-1)\left\{\left\lceil\log _{2} L\right\rceil+1\right\}$ switches are required, where $\lceil x\rceil$ denotes the smallest integer not less than $x$. In addition he has shown that the reconfiguration time of his tunable receiver is very fast and can be as low as $20 \mathrm{~ns}$.

\section{B. Optical CDMA Protocol}

We impose the following assumptions in our model for optical CDMA protocol:

- time is slotted with slot size $T_{s}$;

- messages arrive to a station (or node) with probability $A$, also called users activity;

- message is composed of a fixed number of packets, $\ell$;

- packet is composed of $K$ bits. One packet should fit in a time slot; thus the packet time slot $T_{s}=K L T_{c}$, where $L$ is the code length and $T_{c}$ is the chip time duration;

- each node has a single buffer to store a message; any arrival to a nonempty buffer is disregarded;

- nonempty buffer is freed once the stored message is transmitted successfully;

- node that has a message to transmit, first transmit a connection request to the destination station;

- receiver of an idle station scans across all codes for connection requests in a round-robin (or polling) manner;

- at any time slot, if there is a connection request at a certain code, the station proceeds to send an acknowledgment;

- if there is no requests and there is a message arrival, the station tunes its receiver to the destination code and transmits a connection request on its signature code;

- once an acknowledgment is received, the station proceeds to transmit its message packet by packet (each packet occupies a time slot);

- every time a packet is received successfully at the destination, the receiver transmits an acknowledgment;

- transmitting station follows a go-back $n$ protocol for its transmission with $n$ depending on the propagation time;

- two-way propagation time is assumed to be equal to $t$ time slots;

- transmission times for requests and acknowledgment data are neglected.

\section{Chip-Level Receiver}

Because of its superiority over the correlation model, we assume that the decision rule of any receiver depends on the chip-level model [9], [17]. That is, a data bit 1 is decided if the number of pulses in each weighted chip $Z_{i}$, $i \in \mathcal{X}=\{1,2, \ldots, w\}$, of a code is nonzero [9]. Otherwise, a data bit 0 is declared. Assuming that during a given time slot there are $r \in\{1,2, \ldots, N\}$ active users, we define $\kappa \in\{0,1, \ldots, r-1\}$ and $m \in\{0,1, \ldots, r-1-\kappa\}$ as the number of users that interfere with the desired user at exactly one chip and $w$ chips, respectively. Furthermore, let $\kappa_{i}, i \in \mathcal{X}$ denotes the number of users (out of $\kappa$ users) that interfere with weighted chip $i$. Of course $\kappa=\sum_{i=1}^{w} \kappa_{i}$. We have shown in [17] that the packet success probability for $r \in\{1,2, \ldots, N\}$ active users is given by

$$
\begin{aligned}
P_{s}(r)= & \sum_{\kappa=0}^{r-1} \sum_{m=0}^{r-1-\kappa} \frac{(r-1) !}{\kappa ! m !(r-1-m-\kappa) !} \\
& \cdot p_{1}^{\kappa} p_{w}^{m}\left(1-p_{1}-p_{w}\right)^{r-1-\kappa-m} \\
& \cdot \sum_{\substack{\kappa_{1}, \kappa_{2}, \ldots, \kappa_{w}: \\
\kappa_{1}+\ldots+\kappa_{w}=\kappa}} \frac{\kappa !}{\kappa_{1} ! \ldots \kappa_{w} !} \cdot\left(\frac{1}{w}\right)^{\kappa} \\
& \cdot\left[\frac{1}{2}+\frac{1}{2^{m+1}}\left(\sum_{i=1}^{w} \frac{1}{2^{\kappa_{i}}}-\sum_{i=1}^{w-1} \sum_{j=i+1}^{w} \frac{1}{2^{\kappa_{i}+\kappa_{j}}}\right.\right. \\
& \left.\left.+\ldots+(-1)^{w-1} \frac{1}{2^{\kappa}}\right)\right]^{K}
\end{aligned}
$$

where $p_{1}$ and $p_{w}$ denote the probability of 1 and $w$ chip-interferences, respectively, between two users

$$
\begin{aligned}
& p_{w}=\frac{1}{L} \cdot \frac{1}{|C|}=\frac{1}{L} \cdot\left\lfloor\frac{L-1}{w(w-1)}\right\rfloor^{-1} \\
& p_{1}=\frac{\omega^{2}}{L}-w p_{w} .
\end{aligned}
$$

Because of the round-robin scanning property of the receiver and the go back $n$ (which also mimics a round robin) retransmission property of the transmitter, we call our proposed protocol a round-robin receiver/transmitter (or simply $R^{3} T$ ) random-access protocol.

The remainder of this paper is organized as follows. In Section II, we introduce a mathematical model and a basic description of the state diagram of the proposed protocol. Section III is devoted for a theoretical study for the performance of the proposed protocol, where derivations of both the steady-state system throughput and average packet delay are given. In our analysis, focus is oriented toward multiple access interference only, where the effect of both receivers's shot and thermal noises are neglected. Section IV is maintained for a numerical study of the derived performance measures taking into account the effect of changing several design parameters. Finally, we give our conclusion in Section V.

\section{MATHEMATICAL MODEL}

The state diagram of the $\mathrm{R}^{3} \mathrm{~T}$ protocol is shown in Fig. 1. Each state is labeled by its number of users. The states are defined as follows.

- Initial state $\{m\}$ : A user is in state $m$ if its receiver is scanning across the codes. It takes one time slot to tune to a particular code. Once tuned to a particular code, if the station finds a request on that code (an event that occurs with probability $\sigma$ ), it proceeds to send an acknowledgment. If there is no request and there is an arrival (that occurs with probability $A$ ), the station enters the requesting mode. If there is neither a request nor an arrival, the station remains as is. 


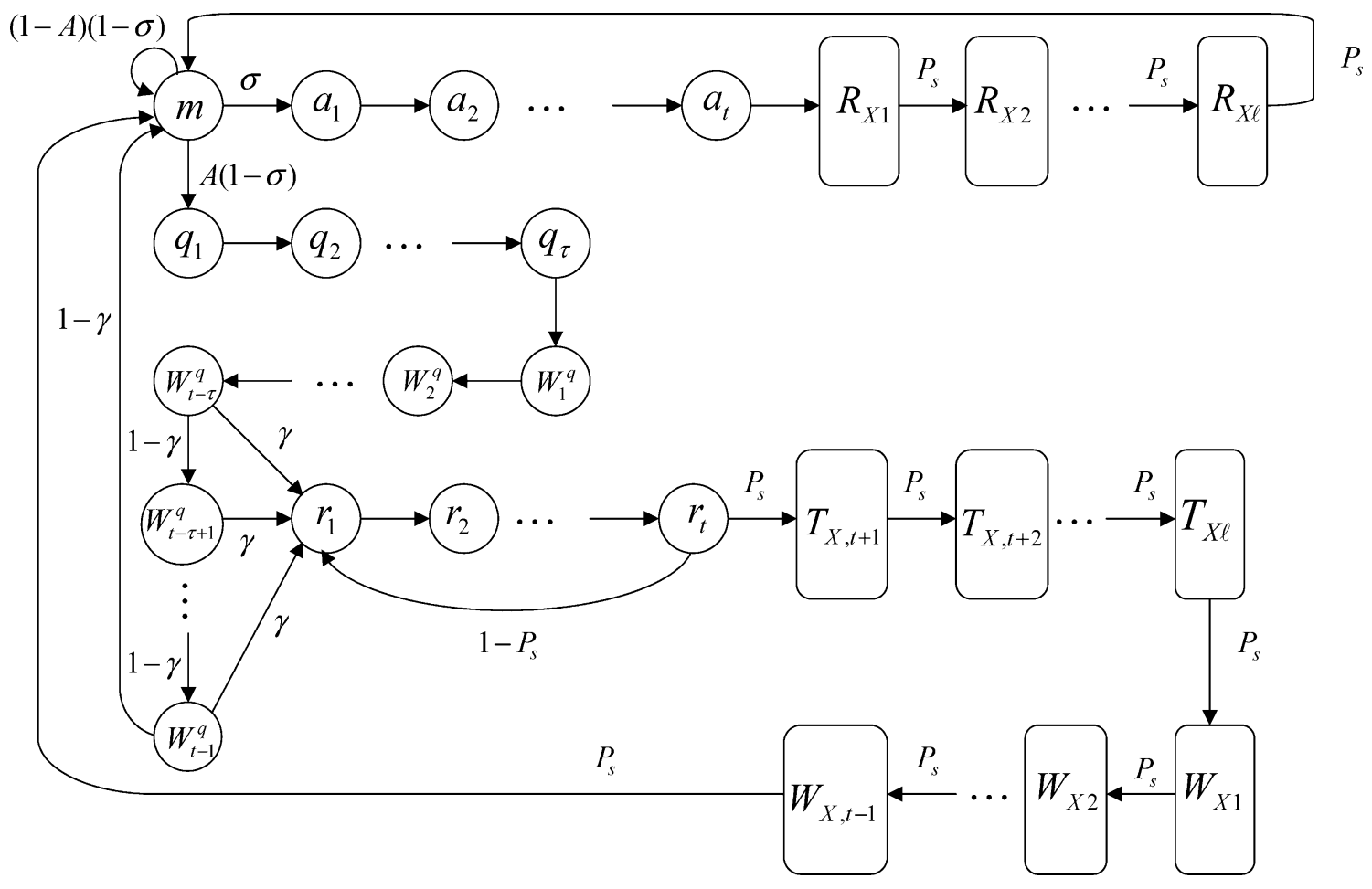

Fig. 1. State diagram of the proposed $\mathrm{R}^{3} \mathrm{~T}$ optical CDMA protocol.

- Requesting mode $\left\{q_{1}, q_{2}, \ldots, q_{\tau}\right\}$ : If there is a message arrival and no request, the station proceeds to send a connection request and tune its receiver to the code of the destination. It takes one time slot to tune a receiver $q_{1}$. At the same time, the station sends repeated requests $\left\{q_{1}, q_{2}, \ldots q_{\tau}\right\}$ for $\tau$ time slots, where $\tau \geq 1$ is the timeout duration in time slots. After sending the last request, the station enters a waiting mode $\left\{W_{1}^{q}, W_{2}^{q}, \ldots, W_{t-1}^{q}\right\}, t \geq \tau$, for $t-1$ time slots, until the reception of an acknowledgment from the destination. At the earliest, the station receives an acknowledgment (with probability $\gamma$ ) after a two-way propagation delay of $t$ time slots from the first request $q_{1}$. In this case, the station enters the transmission mode. Otherwise it stays in the waiting mode for the remaining $\tau-1$ time slots. During each slot in the waiting mode, the station may receive an acknowledgment with probability $\gamma$. If no acknowledgment is received, the station is timed out and goes back to the initial state $m$.

- Acknowledgment mode $\left\{a_{1}, a_{2}, \ldots, a_{t}\right\}$ : The station enters these states after the arrival of a connection request. The station sends an Acknowledgment to the requesting station and waits for $t$ time slots until it starts to receive the first packet. At this instant it enters the reception mode.

- Reception mode $\left\{R_{X 1}, R_{X 2}, \ldots, R_{X \ell}\right\}$ : The station enters state $s_{i}$ in the set of states $R_{X i}$, Figs. 1 and 2(a), if it starts receiving packet $i, i \in\{1,2, \ldots, \ell\}$. If the packet is received successfully, the station sends an acknowledgment to the transmitting station and moves to state $s_{i+1}$ in the set $R_{X, i+1}$. if the packet is damaged, however, the station sends an ask-for-retransmission and enters a waiting mode $\left\{W_{1}^{s i}, W_{2}^{s i}, \ldots, W_{t-1}^{s i}\right\}$ in the set $R_{X, i}$, Fig. 2(a), until the arrival of the retransmitted packet $i$. Finally, if

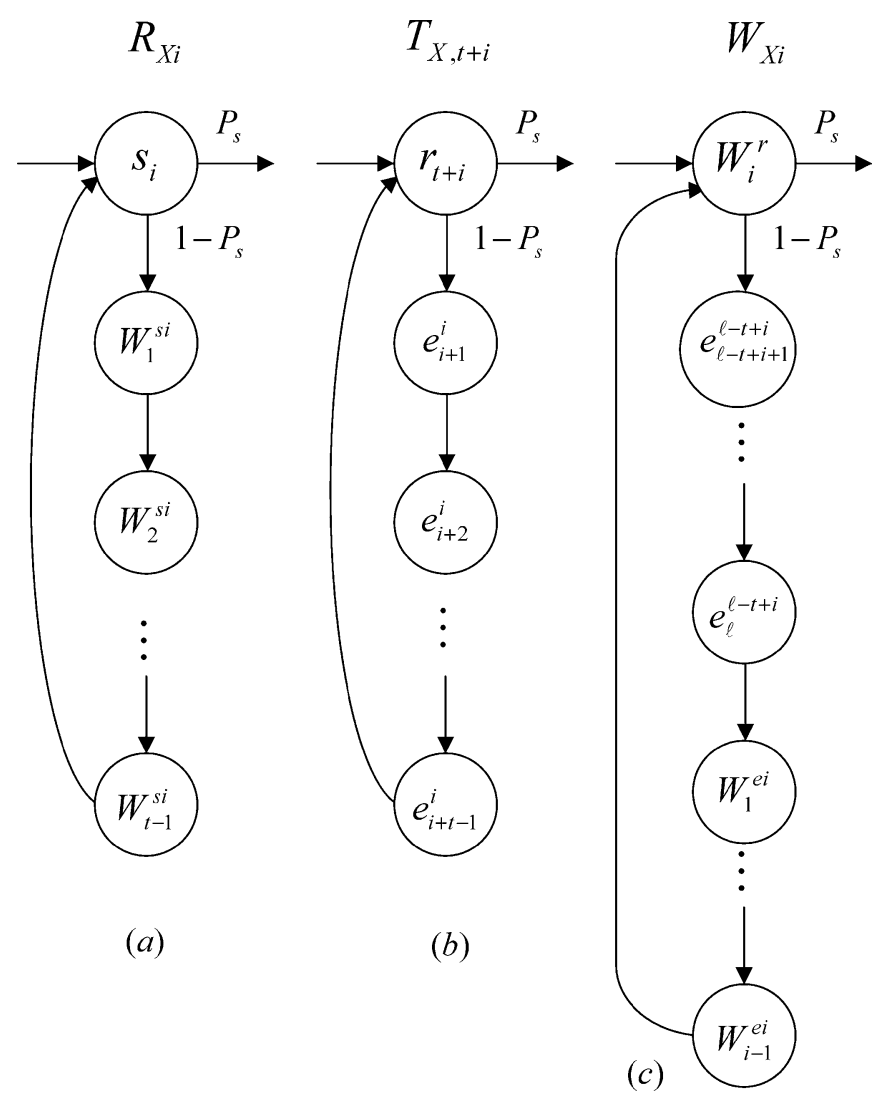

Fig. 2. (a) State $R_{X i}$. (b) State $T_{X, t+i}$. (c) State $W_{X i}$.

the station is receiving the final packet (i.e., it is in state $s_{\ell}$ of set $R_{X, \ell}$ ) and the packet is received successfully, it goes back to the initial state $m$ 
- Transmission mode, $\left\{r_{1}, r_{2}, \ldots, r_{t}, T_{X, t+1}, T_{X, t+2}\right.$, $\left.\ldots, T_{X \ell}\right\}$. The station is in state $r_{i}, i \in\{1,2, \ldots, t\}$, if it is transmitting the first $t$ packets, where $t<\ell$. After transmitting packet number $t$, the station will receive an acknowledgment about the status of its first packet (since the two-way propagation time is $t$ ). If the status indicates a successful transmission, the station transmits the next packet (enters state $r_{t+1}$ in the set of states $T_{X, t+1}$ ), Figs. 1 and 2(b). Otherwise, the station goes back to state $r_{1}$ and start retransmitting the whole previous $t$ packets. If the station is in state $r_{t+i}$ of set $T_{X, t+i}$, $i \in\{1,2, \ldots, \ell-t\}$, Figs. 1 and 2(b), it is transmitting packet $t+i$. After this transmission, it receives a status acknowledgment about packet $i+1$. If the status indicates a successful transmission, the station enters state $r_{t+i+1}$ in set $T_{X, t+i+1}$. Otherwise, it retransmits the last $t$ packets (starting \{from packet $i+1$ ). That is, it enters states $\left\{e_{i+1}^{i}, e_{i+2}^{i}, \ldots, e_{i+t-1}^{i}, r_{t+i}\right\}$ in Fig. 2(b) ( $e$ denotes a packet retransmission). If the station is transmitting the last packet $\ell$ (state $r_{\ell}$ in set $T_{X \ell}$ ) and transmission $\ell-t+1$ is successful, it enters a waiting mode, $\left\{W_{X 1}, W_{X 2}, \ldots, W_{X, t-1}\right\}$, Figs. 1 and 2(c), to collect the status of the last $t-1$ packets. During this waiting mode, if a packet is unsuccessfully received, the station retransmits it and all subsequent packets and waits until a new status is received. That is, it enters the states shown in Fig. 2(c) ( $e$ denotes a packet retransmission and $W$ denotes a waiting slot).

\section{THEORETICAL ANALYSIS}

Because of the complexity of the mathematical model given above, we use the method of equilibrium point analysis to measure the performance of the proposed protocol [18]-[20]. At an equilibrium point of the system, the expected number of users entering a state (in any time slot) is equal to that departing from it. Using this method of analysis, we will be able to figure out the steady-state performance of the system by writing down the flow equation for each state. We start our analysis by assuming that $\ell \geq t$, where $t \geq \tau \geq 1$.

\section{A. Transmission Mode}

This mode involves states $\left\{r_{1}, r_{2}, \ldots, r_{t}\right\}$ and set of states $\left\{T_{X, t+1}, T_{X, t+2}, \ldots, T_{X \ell}\right\}$. From Fig. 1, we have the following flow equations:

$$
r_{1}=r_{2}=\ldots=r_{t}
$$

From Fig. 2, we can write the flow equations for each state in $T_{X, t+i}, i \in\{1,2, \ldots, \ell-t\}$

$$
\begin{aligned}
r_{t+i}= & P_{s} r_{t+i-1}+\left(1-P_{s}\right) r_{t+i} \\
& \Rightarrow r_{t+i}=r_{t+i-1} \Rightarrow r_{t+i}=r_{1} \\
e_{i+1}^{i}= & e_{i+2}^{i}=\ldots=e_{i+t-1}^{i} \\
= & \left(1-P_{s}\right) r_{t+i}=\left(1-P_{s}\right) r_{1} .
\end{aligned}
$$

Similarly, for the states in $W_{X i}, i \in\{1,2, \ldots, t-1\}$

$$
\begin{aligned}
W_{i}^{r} & =r_{1} \\
e_{\ell-t+i+1}^{\ell-t+i} & =e_{\ell-t+i+2}^{\ell-t+i}=\ldots=e_{\ell}^{\ell-t+i}=\left(1-P_{s}\right) r_{1} \\
W_{1}^{e i} & =W_{2}^{e i}=\ldots=W_{i-1}^{e i}=\left(1-P_{s}\right) r_{1} .
\end{aligned}
$$

We define the following variables:

$$
\begin{aligned}
& r \stackrel{\text { def }}{=} \sum_{i=1}^{\ell} r_{i} \\
& W^{r} \stackrel{\text { def }}{=} \sum_{i=1}^{t-1} W_{i}^{r} \\
& e \stackrel{\text { def }}{=} \sum_{i=1}^{\ell-t} \sum_{j=i+1}^{i+t-1} e_{j}^{i}+\sum_{i=1}^{t-1} \sum_{j=\ell-t+i+1}^{\ell} e_{j}^{\ell-t+i} \\
& W^{e} \stackrel{\text { def }}{=} \sum_{i=1}^{t-1} \sum_{j=1}^{i-1} W_{j}^{e i} .
\end{aligned}
$$

Performing the above summations, which involve arithmetic series, we obtain

$$
\begin{aligned}
r & =\ell r_{1} \\
W^{r} & =(t-1) r_{1} \\
e & =\left(1-P_{s}\right)(t-1)\left(\ell-\frac{t}{2}\right) r_{1} \\
W^{e} & =\left(1-P_{s}\right)(t-1)\left(\frac{t}{2}-1\right) r_{1} .
\end{aligned}
$$

\section{B. Reception Mode}

We start by noticing that the number of users receiving packet $i, i \in\{1,2, \ldots, \ell\}$ should be equal to that transmitting packet $i$

$$
s_{i}= \begin{cases}r_{i} P_{s}^{i-1}+e_{i}^{1} P_{s}^{i-2} & \\ +e_{i}^{2} P_{s}^{i-3}+\ldots+e_{i}^{i-1}, & \text { if } i \in\{1,2, \ldots, t\} \\ r_{i} P_{s}^{t-1}+e_{i}^{i+t+1} P_{s}^{t-2} & \\ +e_{i}^{i-t+2} P_{s}^{t-3}+\ldots+e_{i}^{i-1}, & \text { if } i \in\{t+1, t+2, \ldots, \ell\}\end{cases}
$$

Substituting for each $e_{i}^{j}$ and carrying out the summations, which involve geometric series, we get

$$
s_{1}=s_{2}=\ldots=s_{\ell}=r_{1} .
$$

Next we write the flow equations for each state in $R_{X i}, i \in$ $\{1,2, \ldots, \ell\}$

$$
W_{1}^{s i}=W_{2}^{s i}=\ldots=W_{t-1}^{s i}=\left(1-P_{s}\right) r_{1} .
$$

We define the following two variables:

$$
\begin{aligned}
s & \stackrel{\text { def }}{=} \sum_{i=1}^{\ell} s_{i}=\ell r_{1} \\
W^{s} & \stackrel{\text { def }}{=} \sum_{i=1}^{\ell} \sum_{j=1}^{t-1} W_{j}^{s i}=\left(1-P_{s}\right)(t-1) \ell r_{1} .
\end{aligned}
$$




\section{Acknowledgment Mode}

We write the flow equations for the states in the acknowledgment mode

$$
\begin{aligned}
& s_{1}=a_{t}+\left(1-P_{s}\right) s_{1} \Rightarrow a_{t}=P_{s} s_{1}=P_{s} r_{1} \\
& a_{1}=a_{2}=\ldots=a_{t}=\sigma m=P_{s} r_{1} .
\end{aligned}
$$

Hence

$$
m=\frac{P_{s}}{\sigma} r_{1}
$$

Let us define

$$
a \stackrel{\text { def }}{=} \sum_{i=1}^{t} a_{i}=P_{s} t r_{1}
$$

\section{Requesting Mode}

Again by writing the flow equations for the states in this mode, we get

$$
\begin{aligned}
q_{1} & =q_{2}=\ldots=q_{\tau}=A(1-\sigma) m \\
W_{1}^{q} & =W_{2}^{q}=\ldots=W_{t-\tau}^{q}=q_{\tau} \\
W_{t-\tau+i}^{q} & =(1-\gamma)^{i} W_{t-\tau}^{q}, \quad i \in\{1,2, \ldots, \tau-1\} .
\end{aligned}
$$

We define

$$
\begin{aligned}
q+W^{q} & \stackrel{\text { def }}{=} \sum_{i=1}^{\tau} q_{i}+\sum_{i=1}^{t-1} W_{i}^{q} \\
& =\left[t-1+\frac{1}{\gamma}\left(1-(1-\gamma)^{\tau}\right)\right] A \frac{1-\sigma}{\sigma} P_{s} r_{1} .
\end{aligned}
$$

Here, $\gamma$ denotes the probability that a station (in the requesting mode) gets an acknowledgment. It can be evaluated by writing the flow equation into state $r_{1}$

$$
\begin{aligned}
r_{1} & =\gamma \sum_{i=0}^{\tau-1} W_{t-\tau+i}^{q}+\left(1-P_{s}\right) r_{1} \\
& =\gamma \frac{1-\sigma}{\sigma} A P_{s} r_{1} \sum_{i=0}^{\tau-1}(1-\gamma)^{i}+\left(1-P_{s}\right) r_{1} .
\end{aligned}
$$

Hence

$\sigma=A(1-\sigma)\left[1-(1-\gamma)^{\tau}\right] \Rightarrow \gamma=1-\left[1-\frac{\sigma}{A(1-\sigma)}\right]_{(9)}^{\frac{1}{\tau}}$.

The probability that a request is found by a scanning user $\sigma$ is equal to the probability that another user is in states $q_{1}, q_{2}, \ldots$ or $q_{\tau}$

$$
\sigma=\frac{1}{N} \sum_{i=1}^{\tau} q_{i}=\frac{1-\sigma}{\sigma} A P_{s} \tau \frac{r_{1}}{N}, \quad t \geq \tau \geq 1 .
$$

This is a second-order equation whose solution is easily found

$$
\sigma=\frac{1}{2}\left[\sqrt{u^{2}+4 u}-u\right], \quad \text { where } \quad u=A P_{s} \tau \frac{r_{1}}{N} .
$$

\section{E. Steady-State Throughput}

The steady-state throughput $\beta(N, A, t, \tau, \ell)$ is defined as the number of successfully received packets per slot

$$
\beta(N, A, t, \tau, \ell)=\sum_{i=1}^{\ell} s_{i} P_{s}=P_{s}\left(r_{0}\right) \ell r_{1}
$$

where $r_{0}$ denotes the number of transmitting users in a given slot

$$
r_{0}=r+e=\left[\ell+\left(1-P_{s}\right)(t-1)\left(\ell-\frac{t}{2}\right)\right] r_{1} .
$$

Thus

$$
\beta(N, A, t, \tau, \ell)=\frac{P_{s}\left(r_{0}\right) \ell r_{0}}{\ell+\left(1-P_{s}\left(r_{0}\right)\right)(t-1)\left(\ell-\frac{t}{2}\right)} .
$$

Here, $r_{0}$ is determined so as the total number of users in all states is equal to $N$

$$
\begin{aligned}
N=m+r+e+W^{r}+W^{e}+s+W^{s}+a+q+W^{q} \\
=r_{1}\left[2 t \ell\left(1-P_{s}\right)+(2 t+2 \ell-1) P_{s}+\frac{P_{s}}{\sigma}\right. \\
\left.\quad+A(t-1) \frac{1-\sigma}{\sigma} P_{s}+\frac{P_{s}}{1-\left[1-\frac{\sigma}{A(1-\sigma)}\right]^{\frac{1}{\tau}}}\right]
\end{aligned}
$$

where we have used (4)-(9). Substituting for $r_{1}$ from (12), we get

$$
\begin{aligned}
N\left[\ell+\left(1-P_{s}\left(r_{0}\right)\right)(t-1)\left(\ell-\frac{t}{2}\right)\right] \\
=r_{0}\left[2 t \ell\left(1-P_{s}\left(r_{0}\right)\right)+(2 t+2 \ell-1) P_{s}\left(r_{0}\right)\right. \\
+\frac{P_{s}\left(r_{0}\right)}{\sigma}+A(t-1) \frac{1-\sigma}{\sigma} P_{s}\left(r_{0}\right) \\
\left.+\left\{1-\left[1-\frac{\sigma}{A(1-\sigma)}\right]^{\frac{1}{\tau}}\right\}^{-1} P_{s}\left(r_{0}\right)\right]
\end{aligned}
$$

where

$$
\begin{aligned}
& \sigma=\sigma\left(r_{0}\right)=\frac{1}{2}\left[\sqrt{u^{2}+4 u}-u\right] \\
& u=\frac{A P_{s}\left(r_{0}\right) \tau r_{0}}{N\left[\ell+\left(1-P_{s}\left(r_{0}\right)\right)(t-1)\left(\ell-\frac{t}{2}\right)\right]} .
\end{aligned}
$$

That is, $r_{0}$ is the solution of (14).

As mentioned earlier, the above analysis has been performed under the assumption that $\ell \geq t$. The case for $\ell \leq t$ can be analyzed similarly with slight modification. We have the following general result which includes both cases:

$$
\beta(N, A, t, \tau, \ell)=\frac{P_{s}\left(r_{0}\right) \ell r_{0}}{\ell+\left[1-P_{s}\left(r_{0}\right)\right](t \wedge \ell-1)\left(\ell-t \wedge \frac{\ell}{2}\right)}
$$


where $t \wedge \ell \stackrel{\text { def }}{=} \min \{t, \ell\}$ and $r_{0}$ is such that

$$
\begin{aligned}
N\left[\ell+\left(1-P_{s}\left(r_{0}\right)\right)(t \wedge \ell-1)\left(\ell-t \wedge \frac{\ell}{2}\right)\right] \\
=r_{0}\left[2 t \ell\left(1-P_{s}\left(r_{0}\right)\right)+(2 t+2 \ell-1) P_{s}\left(r_{0}\right)\right. \\
+\frac{P_{s}\left(r_{0}\right)}{\sigma}+A(t-1) \frac{1-\sigma}{\sigma} P_{s}\left(r_{0}\right) \\
\left.+\left\{1-\left[1-\frac{\sigma}{A(1-\sigma)}\right]^{\frac{1}{\tau}}\right\}^{-1} P_{s}\left(r_{0}\right)\right]
\end{aligned}
$$

where

$$
\begin{aligned}
\sigma & =\sigma\left(r_{0}\right) \\
& =\frac{1}{2}\left[\sqrt{u^{2}+4 u}-u\right] \\
u & =\frac{A P_{s}\left(r_{0}\right) \tau r_{0}}{N\left[\ell+\left(1-P_{s}\left(r_{0}\right)\right)(t \wedge \ell-1)\left(\ell-t \wedge \frac{\ell}{2}\right)\right]} .
\end{aligned}
$$

\section{F. Protocol Efficiency and Average Delay}

We define here two more performance measures that are variants of the steady-state throughput.

1) Protocol efficiency: It is defined as the fraction of successfully received packets (see (19) at bottom of page). The $1 / 2$ in the denominator of the last fraction is because a transmitter has to find a receiver in order to complete its transmission.

2) Average delay: From Little's theorem, the average packet delay $D$ can be calculated from

$$
D=\frac{N A}{\beta(N, A, t, \tau, \ell)} \quad \text { slots }
$$

where $N A$ is the average offered traffic.

\section{G. Asymptotic Throughput and Efficiency}

For convenience and sake of comparison we derive in this subsection limiting values of the throughput and efficiency as $\ell \rightarrow \infty$. We start by noticing that for $\ell$ large enough, we have from (17) and (18)

$$
r_{0} \rightarrow \frac{N}{2}
$$

and

$$
u \rightarrow \frac{A P_{s} \tau r_{0}}{N \ell\left[\left(1-P_{s}\right) t+P_{s}\right]}, \quad \sigma \rightarrow \sqrt{u}
$$

respectively. Substituting in (16) and (19), we get

$$
\lim _{\ell \rightarrow \infty} \beta(N, A, t, \tau, \ell)=\frac{\frac{N}{2} P_{s}\left(\frac{N}{2}\right)}{\left[\left(1-P_{s}\left(\frac{N}{2}\right)\right)\right] t+P_{s}\left(\frac{N}{2}\right)}
$$

and

$$
\lim _{\ell \rightarrow \infty} \eta=\frac{P_{s}\left(\frac{N}{2}\right)}{\left[\left(1-P_{s}\left(\frac{N}{2}\right)\right)\right] t+P_{s}\left(\frac{N}{2}\right)}
$$

respectively. We notice that the limiting throughput is independent of both the activity $A$ and timeout duration $\tau$. This is because users are busy transmitting (or receiving) their long messages and cannot accept any more arrivals or send any more requests. Furthermore, the asymptotic number of users transmitting at any given slot is indeed $N / 2$. In fact, for any $A>0$, messages keep arriving to new users, who start transmitting and never stop. After a number of slots (large enough), half of the users have long messages being transmitted and the other half are receiving.

\section{H. An Achievable Efficiency}

We have shown in [9] that the bit error rate of chip-level receivers can approach $10^{-9}$ very easily with suitable combinations of code weight, code length, and number of users. Hence $P_{s}(N / 2) \approx 1$ is achievable and consequently an asymptotic efficiency of $\approx 1$ is also achievable [cf. (22)].

\section{NUMERICAL RESULTS}

The steady-state system throughput, protocol efficiency, and average packet delay derived above have been evaluated for chip-level receivers with different network parameters. Our results are plotted in Figs. 3-10. A packet size of $K=127$ bits and a code weight of $w=3$ are held fixed in all results. A chip time constraint $T_{c}=0.254 \mathrm{~ns}$ is imposed in all figures but Fig. 7. A code length of $L=31$ is chosen in all figures but Figs. 7-10. The above parameter selection ensures a slot size of $T_{s}=K L T_{c}=1 \mu \mathrm{s}$. Finally a message length of $\ell=15$ packets and a timeout duration of $\tau=1$ slot (or $\tau T_{c}=1 \mu \mathrm{s}$ ) are imposed in all figures, but Figs. 5, 10, and Fig. 6, respectively.

In Fig. 3, the throughput has been plotted versus the average activity for propagation times $t \in\{2,4,6,8\}$ slots (or interstation distances of $z=v T_{s} t / 2 \in\{200,400,600,800\} \mathrm{m}$, where $v \approx 2 \times 10^{8} \mathrm{~m} / \mathrm{s}$ is the speed of light inside a fiber). The number of users $N$ is chosen to be 30, which is greater than the available number of codes $|C|=5$. General trends of the curves can be noticed. Indeed, for small propagation delays, the throughput increases as $A$ increases until it reaches a saturation value, whereas for greater values of the propagation

$$
\begin{aligned}
\eta \stackrel{\text { def }}{=} & \frac{\text { Number of successfully received packets }}{\text { Maximum number of packets available for transmission }} \\
& =\frac{\beta(N, A, t, \tau, \ell)}{\frac{N}{2}} .
\end{aligned}
$$




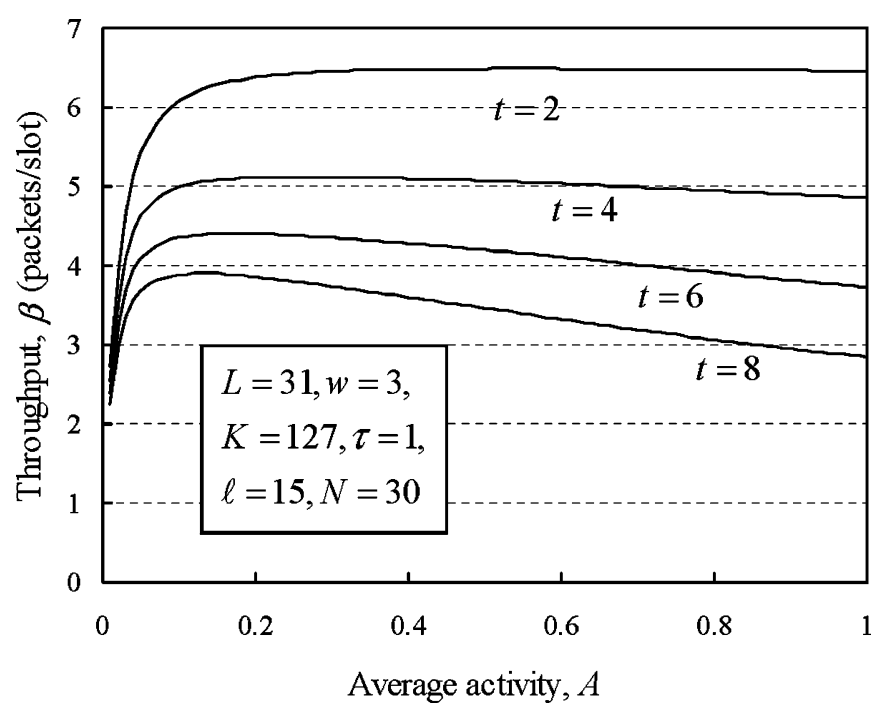

Fig. 3. Throughput versus activity $A$ for different interstation distances.

delays, the throughput increases as $A$ increases until it reaches a maximum value and then decreases when increasing $A$ furthermore. The maximum value reachable by the throughput always occurs at the same value of activity $A$. This value is greater than the number of codes $|C|=5$ for small values of propagation delays. In fact the initial increase of throughput is because as $A$ increases above zero, more packets become available with low interference. The throughput decay in the case of long propagation delays after reaching a peak value is because when $A$ becomes large enough, the number of active users trying to transmit increases, while other users already in the transmission mode are still busy transmitting their messages over a long distance. The interference would thus increase rapidly and packet failures become more probable.

In Fig. 4, the throughput has been plotted versus the number of users $N$ for different propagation times $t \in\{2,4,6\}$ slots and same average activity $A=0.5$. Similar trends of the curves can be noticed. There is always an optimum value of $N$ that maximizes the throughput. Also the peak value changes slightly with the increase in propagation delay. The reason for these bell shapes can be argued as we did in Fig. 3, but the fall edge drops much faster because the increase in the competing users is much larger.

In Fig. 5, we have plotted the throughput versus the message length $\ell$. Again an optimum value of $\ell$ always exists. But the throughput reaches a saturation value as $\ell$ increases without limit. Indeed if users have messages of unlimited length to transmit, every user will be busy either transmitting or receiving packets. In this case, no change in the interference pattern happens. It can be double checked that the limits in Fig. 5 are consistent with the asymptotic results in (21).

In Fig. 6 we have plotted the throughput versus timeout duration $\tau$ for various arrivals (activities). It can be seen that the throughput increases as $\tau$ increases until it reaches a maximum value and then decreases. Indeed as $\tau$ increases, more requests can be acknowledged and $\beta$ increases. By increasing $\tau$ too much, however, the stations are spending too much time in the requesting mode, leaving few stations available to acknowledge requests.

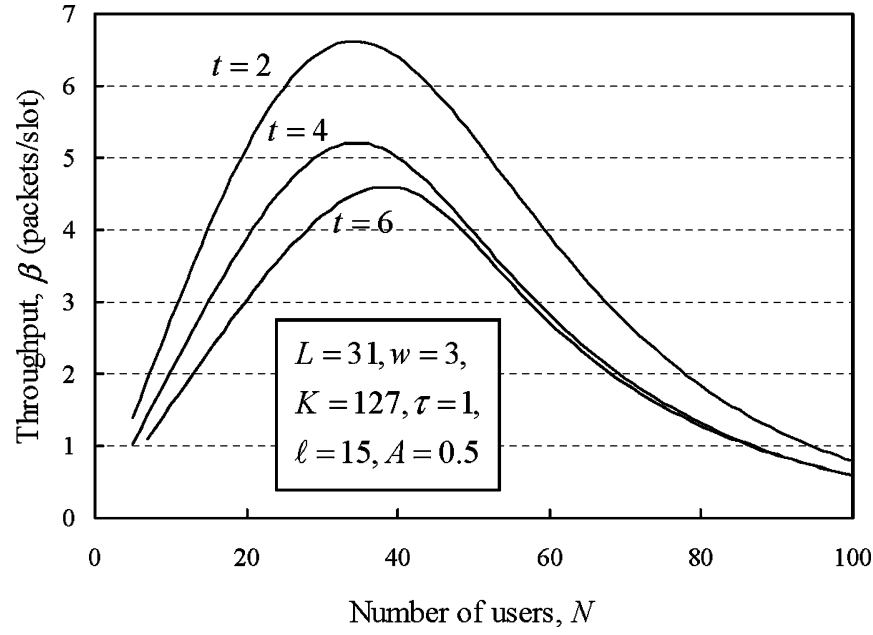

Fig. 4. Throughput versus number of users $N$ for different interstation distances.

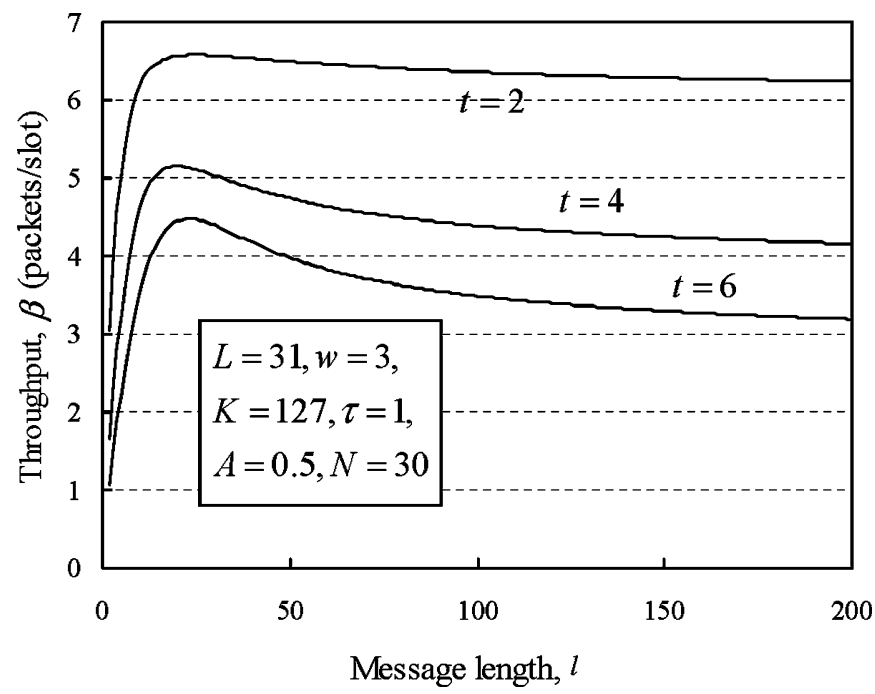

Fig. 5. Throughput versus message length $\ell$ for different interstation distances.

In Figs. 7 and 8, the throughput has been plotted versus $N$ for different code lengths $L$. The difference between the two figures is that in Fig. 7, the slot size is kept fixed at $T_{s}=1 \mu \mathrm{s}$. Thus, the chip time $T_{c}$ should be decreased accordingly. It is obvious that in this case the increase of $L$ leads to a better throughput. Indeed, this increase in $L$ would decrease the possibility of interference patterns, leading to more successful packets and no need to keep retransmission of failed packets. Of course, this advantage is gained at the expense of increasing the system complexity.

In Fig. 8, however, we put back the constraint on the chip time (which is fixed at $T_{c}=0.254 \mathrm{~ns}$ ). Increasing $L$ in this case enforces an increase to the slot size $T_{s}=K L T_{c}$. However, the propagation time $t$ (in slots) will decrease for a fixed interstation distance $z=600 \mathrm{~m}$. In this figure, we use a normalized throughput in packets/ $\mu$ s rather that in packets/slot, since the slot size is no longer fixed. It can be seen from Fig. 8 that for small number of users, it is better to use smaller code lengths and as the number of users increases, longer codes would give better throughput. Indeed, for small number of users, the interference among them is not that much. This leads to almost 


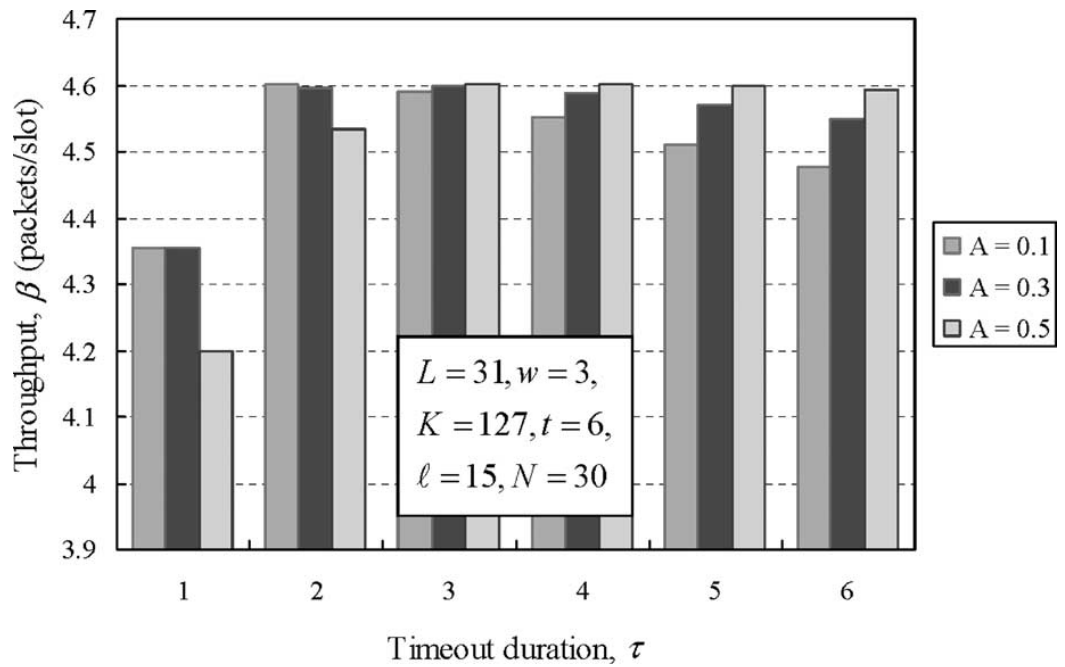

Fig. 6. Throughput versus timeout duration $\tau$ for different activities.

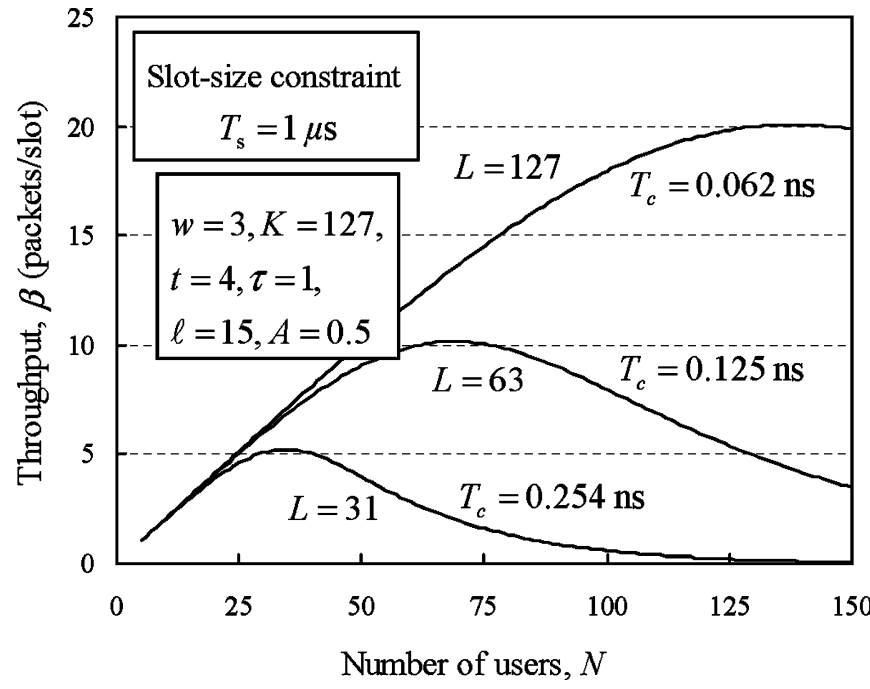

Fig. 7. Throughput versus number of users $N$ for different code lengths and no constraints on chip times.

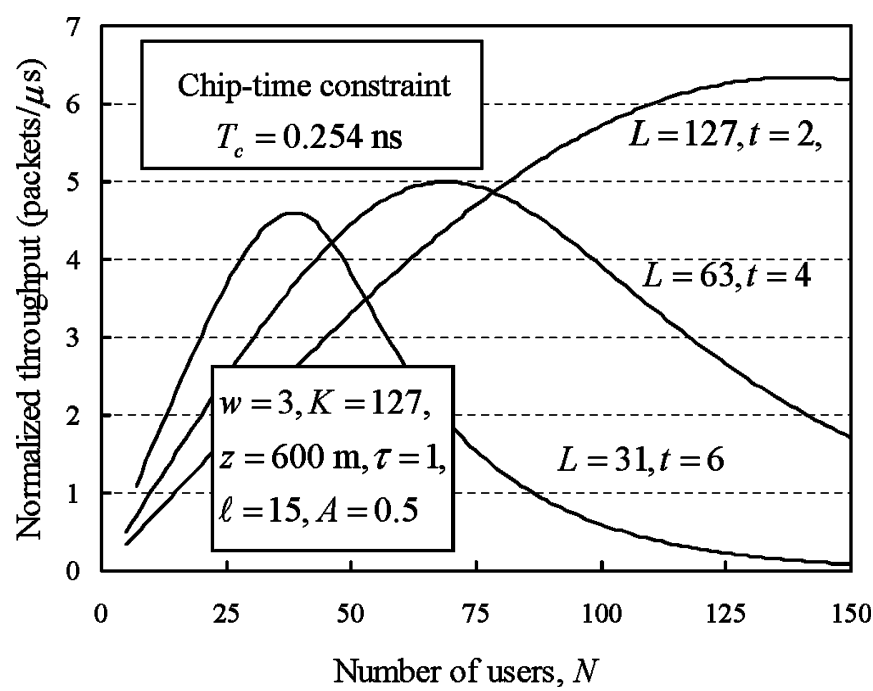

Fig. 8. Throughput versus number of users $N$ for different code lengths and a fixed chip time.

same number of successful packets per slot, independent of the code length. The use of longer codes here increases the slot size

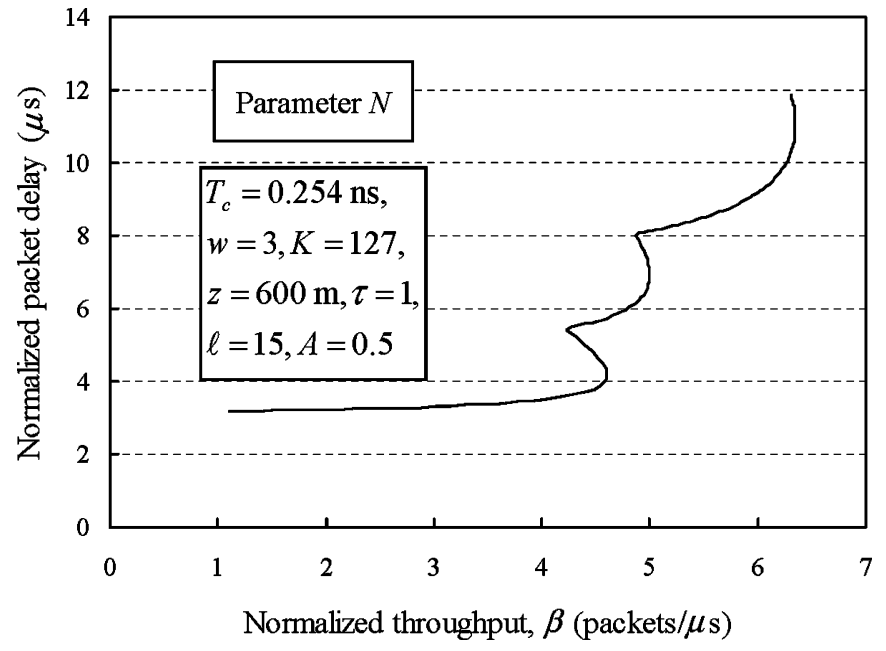

Fig. 9. Normalized packet delay versus normalized throughput for different number of users.

and decreases the number of successful packets per unit time. Thus, it would be better to use shorter codes. However, for large number of users, the interference becomes too much to use short codes. In this case, most of the packets fail during transmission and users become busy retransmitting their failed data. Thus, it would be better to use longer codes.

In Fig. 9 we have plotted normalized packet delays versus normalized throughput for the results in Fig. 8, with $L \in\{31,63,127\}$. The maximum throughput of the three code lengths is selected in our plotting. This explains the zigzag shape of the curve. It is immediate to notice that the high throughput achievement is obtained at the expense of slight increase in the average packet delay. We wish to emphasis that there is an average propagation delay of $2 z / v=6 \mu \mathrm{s}$ between the transmission of a packet and the reception of an acknowledgment.

Finally, in Fig. 10 we have plotted the protocol efficiency versus the message length $\ell$ for different number of users and code lengths. As we have argued in the theoretical analysis, for any given number of stations, the protocol efficiency can approach $100 \%$ with suitable choices of code weight and code length. 


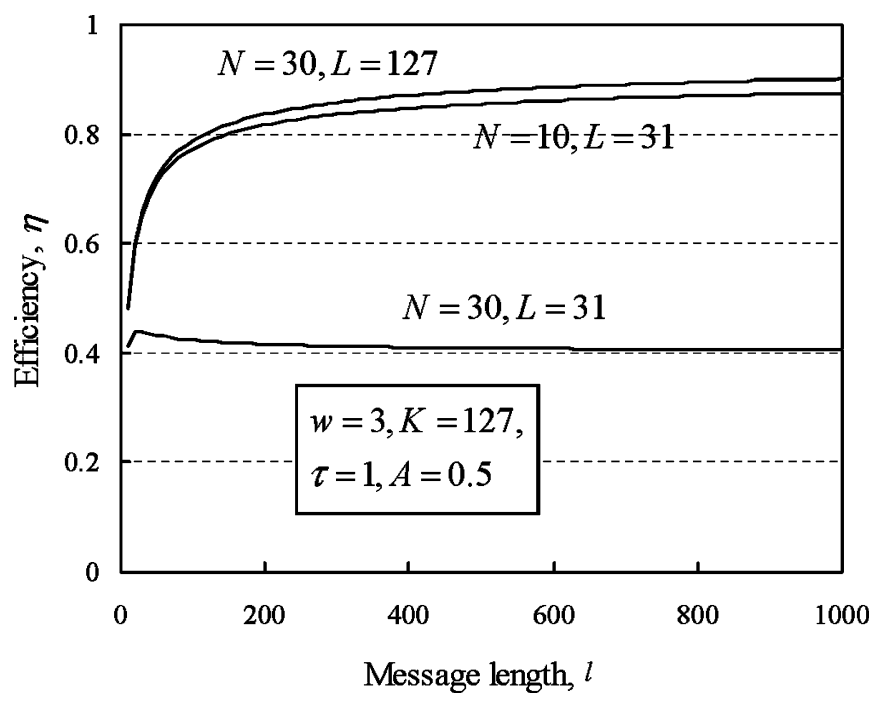

Fig. 10. Protocol efficiency versus message length $\ell$ for different number of users.

\section{CONCLUding REMARKS}

A random-access protocol, called round-robin receiver/transmitter $\left(R^{3} T\right)$ protocol, has been proposed for an optical direct-detection CDMA packet network. Three main performance measures have been derived based on the equilibrium point analysis technique. These measures are the steady-state system throughput, protocol efficiency, and average packet delay. The effect of several design parameters on the system performance measures have been investigated and presented numerically for the case of chip-level receivers. In order to have some insight on the problem under consideration, focus has been given to multiple-access interference only and other sources of noise have been neglected. The following concluding remarks can be extracted from our results.

1) An asymptotic efficiency of $\approx 100 \%$ can be reached easily with suitable choices of code weight and code length.

2) Optimum values (that maximizes the throughput) of the users activity, number of users, and timeout duration always exist.

3) The throughput approaches a saturation value (close to its peak) as the message length increases without limit.

4) For small number of users, better throughput is achieved with short code lengths, whereas for large number of users, better throughput is achieved with longer codes.

5) The proposed protocol exhibits an acceptable average packet delay.

\section{REFERENCES}

[1] F. R. K. Chung, J. A. Salehi, and V. K. Wei, "Optical orthogonal codes: Design, analysis, and applications," IEEE Trans. Inform. Theory, vol. 35, pp. 595-604, May 1989.

[2] J. A. Salehi and C. A. Brackett, "Code division multiple-access techniques in optical fiber networks-Part II: Systems performance analysis," IEEE Trans. Commun., vol. 37, pp. 834-842, Aug. 1989.

[3] R. M. Gagliardi, A. J. Mendez, M. R. Dale, and E. Park, "Fiber-optic digital video multiplexing using optical CDMA," J. Lightwave Technol., vol. 11 , pp. 20-26, Jan. 1993

[4] M. Brandt-Pearce and B. Aazhang, "Performance analysis of single-user and multiuser detectors for optical code division multiple access communication systems," IEEE Trans. Commun., vol. 43, pp. 435-444, Feb./Mar./Apr. 1995.

[5] H. M. H. Shalaby, "Performance analysis of optical synchronous CDMA communication systems with PPM signaling," IEEE Trans. Commun., vol. 43, pp. 624-634, Feb./Mar./Apr. 1995.
[6] J. G. Zhang, "High-speed optical fiber networks using code-division multiple access for future real-time computer communications," IEICE Trans. Commun., vol. E79-B, pp. 932-938, July 1996.

[7] T. Ohtsuki, "Performance analysis of direct-detection optical asynchronous CDMA systems with double optical hard-limiters," $J$. Lightwave Technol., vol. 15, pp. 452-457, Mar. 1997.

[8] - "Direct-detection optical asynchronous CDMA systems with double optical hard-limiters: APD noise and thermal noise," in Proc. IEEE Global Telecommun. Conf. (GLOBECOM'98), Sydney, Australia, Nov. 8-12, 1998, pp. 1616-1621.

[9] H. M. H. Shalaby, "Chip-level detection in optical code-division multiple-access," J. Lightwave Technol., vol. 16, pp. 1077-1087, June 1998.

[10] S. Zahedi and J. A. Salehi, "Analytical comparison of various fiberoptic CDMA receiver structures," J. Lightwave Technol., vol. 18, pp. 1718-1727, Dec. 2000.

[11] T. W. F. Chang and E. H. Sargent, "Optical CDMA using 2-D codes: the optimal single-user detector," IEEE Commun. Lett., vol. 5, pp. 169-171, Apr. 2001.

[12] H. M. H. Shalaby, "Complexities, error probabilities, and capacities of optical OOK-CDMA communication systems," IEEE Trans. Commun., vol. 50, pp. 2009-2015, Dec. 2002.

[13] C.-S. Hsu and V. O. K. Li, "Performance analysis of slotted fiber-optic code-division multiple-access (CDMA) packet networks," IEEE Trans. Commun., vol. 45, pp. 819-828, July 1997.

[14] _ - "Performance analysis of unslotted fiber-optic code-division multiple-access (CDMA) packet networks," IEEE Trans. Commun., vol. 45, pp. 978-987, Aug. 1997.

[15] J. Mückenheim and K. Iversen, "A new design method for optical CDMA computer networks," in Proc. IEEE Symp. Computers and Communications (ISCC '97), Alexandria, Egypt, July 1-3, 1997, pp. 589-593.

[16] A. Stok and E. H. Sargent, "System performance comparison of optical CDMA and WDMA in a broadcast local area network," IEEE Commun. Lett., vol. 6, pp. 409-411, Sept. 2002.

[17] H. M. H. Shalaby, "Optical CDMA random access protocols with and without pretransmission coordination," J. Lightwave Technol., vol. 21, pp. 2455-2462, Nov. 2003.

[18] J. P. Jue, M. S. Borella, and B. Mukherjee, "Performance analysis of the rainbow WDM optical network prototype," IEEE J. Select. Areas Commun., vol. 14, pp. 945-951, June 1996.

[19] C.-T. Chen, Linear System Theory and Design, 3rd ed. New York: Oxford Univ. Press, 1999.

[20] J.-R. Sack and J. Urrutia, Handbook of Computational Geometry. North Holland, The Netherlands: Elsevier, 2000.

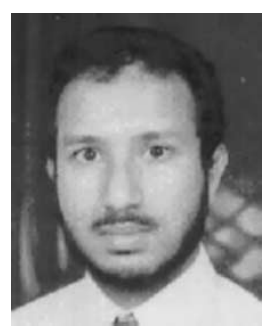

Hossam M. H. Shalaby (S'83-M'91-SM'99) was born in Giza, Egypt, in 1961. He received the B.S. and M.S. degrees from the University of Alexandria, Egypt, in 1983 and 1986, respectively, and the Ph.D. degree from the University of Maryland, College Park, MD, in 1991, all in electrical engineering.

In 1991, he joined the Department of Electrical Engineering, University of Alexandria, Egypt, as an Assistant Professor. He was promoted to the position of Associate Professor in 1996 and then to Professor in 2001 (current position). Since December 2000, he has been an Adjunct Professor with Department of Electrical and Information Engineering, Faculty of Sciences and Engineering, Laval University, Quebec, Canada. From March to April 1996, he was a Visiting Professor at the Electrical Engineering Department, Beirut Arab University, Lebanon. From September 1996 to January 1998, he was an Associate Professor with the Electrical and Computer Engineering Department, International Islamic University Malaysia, and, from February 1998 to December 1998, he was with the School of Electrical and Electronic Engineering, Nanyang Technological University, Singapore, where he was a Senior Lecturer and, from January 1999 to February 2001, an Associate Professor. His research interests include optical communications, optical CDMA, spread-spectrum communications, and information theory.

Dr. Shalaby received the SRC Fellowship from 1987 to 1991 (Systems Research Center, MD), the Shoman Prize for Young Arab Researchers in 2002 (The Abdul Hameed Shoman Foundation, Amman, Jordan), the State Award twice in 1995 and 2001 (Academy of Scientific Research and Technology, Egypt), and the University Award in 1996 (University of Alexandria, Egypt). He has served as a Student Branch Counselor (Alexandria University), IEEE Alexandria and North Delta Subsection, since 2002, and served as a Chairman of the Student Activities Committee of IEEE Alexandria Subsection from 1995 to 1996 . He has also served as a technical referee for Proceedings of the Institution of Electrical Engineers, IEEE TRANSACTIONS ON COMMUNICATIONS, IEEE TRANSACTIONS ON INFORMATION THEORY, IEEE JOURNAL ON SELECTED AREAS IN COMMUNICATIONS, and the JOURNAL OF LightWAVE TECHNOLOGY. He is listed in the 14th edition of Marquis Who's Who in the World, 1997. 